\title{
Modular Strategies for Recursive Game Graphs $^{\star} \star \star$
}

\author{
Rajeev Alur ${ }^{1}$, Salvatore La Torre ${ }^{2}$, and Parthasarathy Madhusudan ${ }^{1}$ \\ 1 University of Pennsylvania \\ 2 Università degli Studi di Salerno
}

\begin{abstract}
In this paper, we focus on solving games in recursive game graphs that can model the control flow in sequential programs with recursive procedure calls. While such games can be viewed as the pushdown games studied in the literature, the natural notion of winning in our framework requires the strategies to be modular with only local memory; that is, resolution of choices within a module does not depend on the context in which the module is invoked, but only on the history within the current invocation of the module. While reachability in (global) pushdown games is known to be EXPTIME-complete, we show reachability in modular games to be NP-complete. We present a fixpoint computation algorithm for solving modular games such that the worst-case number of iterations is exponential in the total number of returned values from the modules. If the strategy within a module does not depend on the global history, but can remember the history of the past invocations of this module, that is, if memory is local but persistent, we show that reachability becomes undecidable.
\end{abstract}

\section{Introduction}

The original motivation for studying games in the context of formal analysis of systems comes from the controller synthesis problem. Given a description of the system where some of the choices depend upon the input and some of the choices represent uncontrollable internal non-determinism, designing a controller that supplies inputs to the system so that the product of the controller and the system satisfies the correctness specification corresponds to computing winning strategies in two-player games. This question has been studied extensively in the literature (see 51510] for sample research and [19] for a survey). Besides the long-term dream of synthesizing correct programs from formal specifications, games are relevant in two different contemporary contexts. First, model checking for branching-time logics such as $\mu$-calculus, as well as several procedures that

* This research was supported in part by ARO URI award DAAD19-01-1-0473, NSF CAREER award CCR97-34115, and NSF award ITR/SY 0121431. The second author was also supported by the MIUR in the framework of the project "Metodi Formali per la Sicurezza" (MEFISTO) and MIUR grant $60 \% 2002$.

$\star \star$ For the details of the proofs of this paper we refer the reader to the technical report available at the URL:"http://www.cis.upenn.edu/ madhusud/". 
use tree automata emptiness for deciding various logics, can be reduced to solving games 918. Second, games have been shown to be relevant for verification of open systems. For instance, the Alternating Temporal Logic allows specification of requirements such as "module A can ensure delivery of the message no matter how module B behaves" [2] module checking deals with the problem of checking whether a module behaves correctly no matter in which environment it is placed [11]; and the framework of interface automata allows assumptions about the usage of a component to be built into the specification of the interface of the component, and formulates compatibility of interfaces using games 8 .

In traditional model checking, the model is a finite state machine whose vertices correspond to states, and whose edges correspond to transitions. To define two-player games in this model, the vertices are partitioned into two sets corresponding to the two players, where a player gets to choose the transition when the current state belongs to its own partition 1 1 . In this paper, we consider the richer system model of recursive state machines (RSMs), in which vertices can either be ordinary states or can correspond to invocations of other state machines in a potentially recursive manner. Recursive state machines can model the control flow in typical sequential imperative programming languages with recursive procedure calls.

More precisely, a recursive state machine consists of a set of component machines called modules. Each module has a set of nodes (atomic states) and boxes (each of which is mapped to a module), a well-defined interface consisting of entry and exit nodes, and edges connecting nodes/boxes. An edge entering a box models the invocation of the module associated with the box, and an edge leaving a box corresponds to a return from that module. To define two-player games on recursive state machines, we partition the nodes into two sets such that a player gets to choose the transition when the current node belongs to its own partition. We focus on solving the reachability game, that is, deciding if one of the players has a strategy to force the system starting from a specified node to enter one of the target nodes.

Due to recursion, the underlying global state-space is infinite and behaves like a pushdown system. While reachability in pushdown games is already studied [206], we are interested in developing algorithms for games on RSMs for two reasons. First, RSMs is a more natural model of recursive systems, and studying reachability (without games) on RSMs has led to refined bounds on complexity in terms of parameters such as the number of entry and exit nodes of modules [1. Second, existing algorithms for solving pushdown games assume that each player has access to the entire global history which includes the information of the play in all modules. The first contribution of the paper is the notion of modular strategies for games on RSMs. A modular strategy is a strategy that has only local memory, and thus, resolution of choices within a module does not depend on the context in which the module is invoked, but only on the history

\footnotetext{
${ }^{1}$ More interesting forms of interactions between the two players are possible, for instance, see alternating transition systems [2], but we will use a simple game model for this paper.
} 
within the current invocation of the module. This permits a natural definition of synthesis of recursive controllers: a controller for a module can be plugged into any context where the module is invoked. Clearly, there are cases where there is no modular strategy, but there is a global one. Recent work on the interface compatibility checking for software modules implements the global games on pushdown systems [7, but we believe that checking for existence of modular strategies matches better with the intuition for compatibility.

After formulating the notion of modular strategies, we show that deciding existence of modular winning strategies for reachability games is NP-complete. In contrast, global reachability games are EXPTIME-complete [20]. Then, we proceed to formulate a fixpoint computation algorithm that generalizes the symbolic solution to reachability games. For ordinary game graphs, the fixpoint algorithm, starting with the target vertices, iteratively grows the set of vertices from which winning is ensured. In our case, when a node is found to be winning, the algorithm also needs to keep track of the strategies within different modules that were used. This labeling is needed to make sure that the same set of module strategies is used consistently everywhere to ensure modularity. It turns out that the only relevant information about a strategy used within a module is the set of exit nodes of the module that the strategy can restrict the game into. Consequently, in the worst-case, the number of iterations of our fixpoint computation is exponential in the number of exit nodes of the modules.

We also consider safety winning conditions for recursive game graphs. Since we restrict to modular strategies of the protagonist, on recursive game graphs safety is not dual to reachability. We prove that determining the existence of a modular strategy for a safety recursive game is also NP-complete.

Finally, we consider the case when the strategy within a module is required to have only local memory, and does not depend on the global history, but this memory can be persistent, and can remember the history of the past invocations of this module. In this case, we prove the reachability games to be undecidable by a reduction from the undecidability of multi-player games with incomplete information [14].

Related work: We have already explained the relation to the global games on pushdown systems $4,6,20]$. The notion of modular strategies may remind the reader of games with partial information, but this is technically quite different from the standard notion of partial information, and, in fact, lowers the complexity class of the decision problem, while introducing partial information typically adds an exponential to the complexity. Another context where modular strategies have been studied is in the realm of concurrent or distributed games where the intention is to come up with distributed controllers for a system (see [16 12 17] and references therein). In that setting, however, looking for modular strategies quickly leads to undecidability. There are some restricted architectures that are decidable, a prominent one being the hierarchic architectures [1416]. Our problem, however, is quite different from these works since in our setting the control is always in one module, while in the concurrent setting, control can be in several modules at any given time. 


\section{Games on Recursive Graphs}

In this section we introduce recursive games and the decision problem we wish to solve. We start recalling the notion of flat games which are the standard games on And-Or graphs.

\subsection{Flat Game Graphs}

A flat game graph is a tuple $G=\left\langle V, V_{0}, V_{1}, \gamma\right\rangle$ where $V$ is a finite set of vertices, $V_{0}$ and $V_{1}$ define a partition of $V$, and $\gamma: V \rightarrow 2^{V}$ is a function giving for each vertex $u \in V$ the set of its successors in $G$. The game is played by two players, player 0 (the protagonist) and player 1 (the adversary). For $p=0,1$, the vertices in $V_{p}$ are those from which only player $p$ can move and the allowed moves are given by the function $\gamma$. A play of a game graph $G$ is a (finite or infinite) path in $G$ constructed in turns by the two players. A play $u_{0} u_{1} \ldots$ starting at a vertex $u_{0}$ is constructed as follows: if $u_{0} \in V_{p}$, player $p$ chooses a successor vertex $u_{1}$; at each step $j$, player $p$ (where $u_{j} \in V_{p}$ ) chooses a successor vertex $u_{j+1}$.

A strategy for player $p$ is a function $f: V^{*} \rightarrow V$ mapping sequences (and hence plays) to vertices. The idea is that when a play $\pi u$ has been played, where $u \in V_{p}$, the strategy $f$ recommends the move $f(\pi u)$. A play $\pi=u_{1} u_{2} \ldots$ is according to $f$ if for every $j<|\pi|$ such that $u_{j} \in V_{p}, f\left(u_{0} \ldots u_{j}\right)=u_{j+1}$. When a strategy for player $p$ depends only on the current vertex of a play, i.e., if $f(\pi x)=f\left(\pi^{\prime} x\right)$, for all plays $\pi, \pi^{\prime}$ and $x \in V_{p}$, it is called a memoryless strategy. A play $\pi$ according to a strategy is said to be maximal if it cannot be continued (i.e. if $\pi$ is infinite or there is no $v \in V$ such that $\pi v$ is a play according to the strategy).

Since we are interested in reachability games in this paper, we have a winning condition for the game given by a subset of target vertices $X$. A flat reachability game is then a tuple $\left\langle G, v_{0}, X\right\rangle$ where $G$ is a flat game graph, $v_{0}$ is the initial vertex where the plays start, and $X$, a subset of the vertices, is the target set. A play is winning for the protagonist if it contains a vertex in $X$ (i.e. a play $\pi=u_{0} u_{1} \ldots$, where $u_{0}=v_{0}$, is winning if there is an $i<|\pi|$ such that $\left.u_{i} \in X\right)$. A strategy for the protagonist is winning if all maximal plays according to it are winning, while a strategy for the adversary is winning if all maximal plays according to it are not winning. Hence, a winning strategy for the protagonist is one which forces the play to the target set $X$, no matter how the adversary chooses. Flat reachability games are PTIME-complete and if a player has a winning strategy, it also has a memoryless winning strategy [13].

\subsection{Recursive Game Graphs}

In this paper, our main objective is to study reachability games in hierarchical and recursive graph structures that are defined using several interacting component game graphs (or game modules). Our model is a generalization to game graphs of the recursive state machines model defined in [1]. 


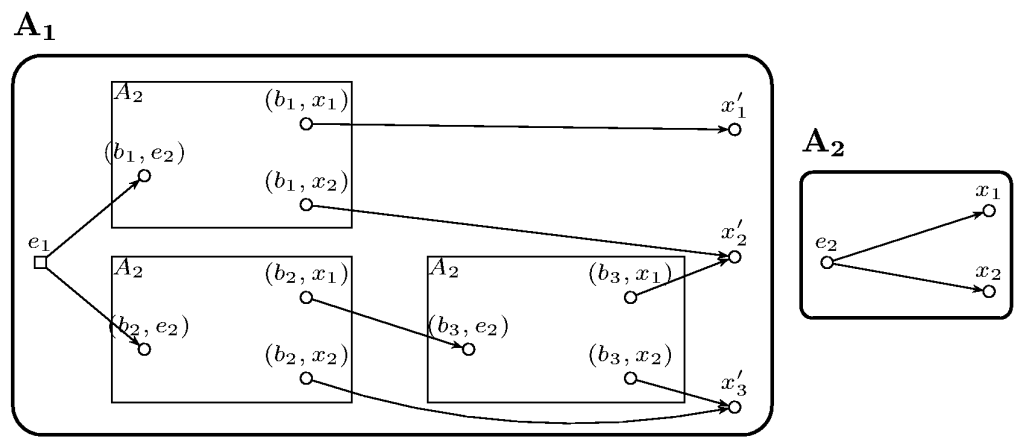

Fig. 1. A recursive game graph

A recursive game graph $A$ is given by a tuple $\left\langle A_{1}, \ldots, A_{n}\right\rangle$, where each game module $A_{i}=\left(N_{i}, B_{i}, V_{i}^{0}, V_{i}^{1}, Y_{i}, E n_{i}, E x_{i}, \delta_{i}\right)$ consists of the following components:

- A set of nodes $N_{i}$ which we call the nodes of module $A_{i}$.

- A nonempty set of entry nodes $E n_{i} \subseteq N_{i}$ and a nonempty set of exit nodes $E x_{i} \subseteq N_{i}$.

- A set of boxes $B_{i}$.

- Two disjoint sets $V_{i}^{0}$ and $V_{i}^{1}$ that partition the set of nodes and boxes into two sets, i.e. $V_{i}^{0} \cup V_{i}^{1}=N_{i} \cup B_{i}$ and $V_{i}^{0} \cap V_{i}^{1}=\emptyset$. The set $V_{i}^{0}\left(V_{i}^{1}\right)$ denotes the places where it is the turn of player 0 (resp. player 1) to play.

- A labeling $Y_{i}: B_{i} \rightarrow\{1, \ldots, n\}$ that assigns to every box an index of the game modules $A_{1}, \ldots, A_{n}$.

- Let Calls $_{i}=\left\{(b, e) \mid b \in B_{i}, e \in E n_{j}, j=Y_{i}(b)\right\}$ denote the set of calls of module $A_{i}$ and let Retns $_{i}=\left\{(b, x) \mid b \in B_{i}, x \in E x_{j}, j=Y_{i}(b)\right\}$ denote the set of returns in $A_{i}$. Then, $\delta_{i}: N_{i} \cup$ Retns $_{i} \rightarrow 2^{N_{i} \cup \text { Calls }_{i}}$ is a transition function.

We also refer to entry (exit) nodes simply as entry (exit). Nodes of $N_{i}$, for any $i$, which are in $V_{i}^{p}$ are called $p$-nodes while returns of the form $(b, u)$, where $b \in V_{i}^{p}$, for some $i$, are called p-returns. An element in Calls $s_{i}$ of the form $(b, e)$ represents a call from $A_{i}$ to the module $A_{j}$, where $j=Y_{i}(b)$ and $e$ is an entry of $A_{j}$. An element in Retns $_{i}$ of the form $(b, x)$ corresponds to the associated return of control from $A_{j}$ to $A_{i}$ when the call exits from $A_{j}$ at exit $x$. The transition function hence defines moves from nodes and returns to a set of nodes and calls.

To illustrate the definitions, consider the example shown in Figure 1 It comprises two modules $A_{1}$ and $A_{2}$, where $A_{1}$ has three boxes that invoke $A_{2}$. The only adversary node is $e_{1}$, the initial node of $A_{1}$.

We make some assumptions of these graphs in the sequel that enable a more readable presentation (these will turn out to be without loss of generality for the problems we consider): 
- There is only one entry point to every module, i.e. $\left|E_{i}\right|=1$ for every $i$. We refer to this unique entry point of $A_{i}$ as $e_{i}$.

- For every $u \in N_{i}, e_{i} \notin \delta_{i}(u)$ holds, and for every $x \in E x_{i}, \delta_{i}(x)$ is empty. That is, there are no transitions from a module to its own entry nodes and no transitions from its exits.

- The nodes and boxes of all agents are disjoint. Let $B=\bigcup_{i} B_{i}$ denote the set of all boxes and $N=\bigcup_{i} N_{i}$ denote the set of all nodes. We extend the functions $\left\{Y_{i}\right\}_{i=1}^{n}$ to a single function $Y: B \rightarrow\{1, \ldots, n\}$.

Note that the above definition allows recursive calls - a module can call itself directly or indirectly. We say that a recursive game graph is hierarchic if this cannot happen. Formally, a recursive game graph $\left\langle A_{1}, \ldots A_{n}\right\rangle$ is hierarchic if there is an ordering $\leq$ on the modules such that for every $A_{i}, A_{i}$ has no calls to any $A_{j}$ where $A_{j} \leq A_{i}$ (i.e. there is no box $b$ in $A_{i}$ such that $A_{Y(b)} \leq A_{i}$ ). For example, the game graph in Figure 1 is hierarchic.

To define the notions of play and strategy for a recursive game graph, we first give the semantics of our model by defining a flat game graph associated with it. This is similar to the way one associates a flat model that describes the behavior of a recursive state machine. A (global) state of a recursive game graph $A=\left\langle A_{1}, \ldots, A_{n}\right\rangle$ is a tuple $\langle\bar{b}, u\rangle$ where $\bar{b}=b_{1}, \ldots, b_{r}$ is a finite (perhaps empty) sequence of boxes from $B$, and $u$ is a node in $N$. Consider a state $\left\langle b_{1}, \ldots, b_{r}, u\right\rangle$ such that $b_{i} \in B_{j_{i}}$ for $1 \leq i \leq r$ and $u \in N_{j}$. Such a state is well-formed if $Y\left(b_{i}\right)=j_{i+1}$ for $1 \leq i<r$, and if $r \geq 1, Y\left(b_{r}\right)=j$. If $\tau$ denotes the empty sequence, note that any state of the form $\langle\tau, u\rangle$ is well-formed; we henceforth use $\langle u\rangle$ to denote $\langle\tau, u\rangle$. Intuitively, a well-formed state $\left\langle b_{1}, b_{2}, u\right\rangle$ denotes the configuration where $b_{1}$ is a call to a module which in turn called another module using $b_{2}$ in which the current node is $u$. When the last module exits, the control goes back to the corresponding return of $b_{2}$, and so on. Henceforth, we assume states to be well-formed and denote by $Q_{A}$ the set of global states of $A$.

According to the partition of nodes and boxes in the recursive game graph, the states are also classified as protagonist and adversary states. For $p=0,1$, a state $\left\langle b_{1}, \ldots, b_{r}, u\right\rangle$ is a $p$-state if either $u$ is not an exit and is a $p$-node, or, $\left(b_{r}, u\right)$ is a $p$-return. (If $r=0$ and $u$ is an exit, the definition will not matter since there are no transitions from it; we hence choose these states to be, say, 0 -states.) We denote by $Q_{p}$ the set of $p$-states.

The global game graph corresponding to a recursive game graph $A$ is $G_{A}=$ $\left(Q_{A}, Q_{0}, Q_{1}, \delta\right)$ where the global transition function $\delta$ is given as follows. Let $s=\left\langle b_{1}, \ldots, b_{r}, u\right\rangle$ be a state with $u \in N_{j}$ and $b_{r} \in B_{m}$. Then, $s^{\prime} \in \delta(s)$ provided one of the following holds:

1. $u^{\prime} \in \delta_{j}(u)$ for a node $u^{\prime}$ of $A_{j}$, and $s^{\prime}=\left\langle b_{1}, \ldots, b_{r}, u^{\prime}\right\rangle$.

2. $\left(b^{\prime}, e\right) \in \delta_{j}(u)$ for a box $b^{\prime}$ of $A_{j}$, and $s^{\prime}=\left\langle b_{1}, \ldots, b_{r}, b^{\prime}, e\right\rangle$.

3. $u$ is an exit node of $A_{j}, u^{\prime} \in \delta_{m}\left(b_{r}, u\right)$ for a node $u^{\prime}$ of $A_{m}$, and $s^{\prime}=$ $\left\langle b_{1}, \ldots, b_{r-1}, u^{\prime}\right\rangle$.

4. $u$ is an exit node of $A_{j},\left(b^{\prime}, e\right) \in \delta_{m}\left(b_{r}, u\right)$ for a box $b^{\prime}$ of $A_{m}$, and $s^{\prime}=$ $\left\langle b_{1}, \ldots, b_{r-1}, b^{\prime}, e\right\rangle$. 
The first case above is when the control stays within the module $A_{j}$, the second case is when a new module is entered via a box of $A_{j}$, the third is when the control exits $A_{j}$ and returns to $A_{m}$, and the last case is when the control exits $A_{j}$ and immediately enters a new module via a box of $A_{m}$. Note that the set of reachable states from, say, a state $\left\langle e_{i}\right\rangle\left(e_{i} \in E n_{i}\right)$ could be infinite if the recursive game graph is not hierarchic (if it is hierarchic, the global reachable graph is finite).

A recursive game is a tuple $\left\langle A, e_{1}, X\right\rangle$ where $A=\left\langle A_{1}, \ldots A_{n}\right\rangle$ is a game graph, $e_{1}$ is the entry node of $A_{1}$, and $X \subseteq E x_{1}$ is the target set, which is a subset of the exits of $A_{1}$. The global recursive game corresponding to $\left\langle A, e_{1}, X\right\rangle$ is the flat game $\left\langle G_{A},\left\langle e_{1}\right\rangle, X^{\prime}\right\rangle$ where $X^{\prime}$ is the set of all global states $s=\left\langle b_{1}, \ldots, b_{r}, u\right\rangle$ where $u \in X$. A (winning) global strategy in a recursive game is a (winning) strategy in the global recursive game.

If we use the notion of global strategies, it will lead us to the definition of games equivalent to pushdown games 20. One can show that for every recursive game, there is a polynomial-sized pushdown automaton whose configuration game graph (as in [20]) is isomorphic to the global graph of the recursive game, and vice-versa. We depart from pushdown games at this point in that we require a particular kind of strategy (namely a modular strategy) that wins these games. In the next section, we introduce this class of strategies and define the corresponding decision problem.

\subsection{Modular Strategies}

We are interested in modular strategies for the protagonist in each game module such that when the strategies are put together, the protagonist wins the game. Such strategies are restricted in that they can only refer to the "local history" of each module, that is, the portion of the play corresponding to the "current" invocation of the module.

To define formally a modular strategy for a recursive game graph $A$, we introduce some notation. For a play $\pi$, the control after $\pi$ is in $A_{i}$ if the current node is in $A_{i}$; however, if the current node is an exit node, then the control is in the module that made the last call. Formally, for a play $\pi s$, we say that the control after $\pi s$ is in $A_{i}$ if $s=\left\langle b_{1}, \ldots, b_{r}, u\right\rangle$, with $u \in N_{i} \backslash E x_{i}$ or $\left(b_{r}, u\right) \in$ Retns $_{i}$. Note that the control after a play can be in at most one module.

Consider a play $\pi=s_{0} s_{1} \ldots s_{k}$ and let the control after $\pi$ be in $A_{i}$. Let $s_{k}=$ $\left\langle b_{1}, \ldots, b_{r}, u\right\rangle$. Then we define the current stack of $\pi$ to be $\beta(\pi)=\left\langle b_{1}, \ldots, b_{r}\right\rangle$, if $u \notin E x$ and $\beta(\pi)=\left\langle b_{1}, \ldots, b_{r-1}\right\rangle$, otherwise. Now, let $j$ be the largest index $0 \leq j \leq k$ such that $s_{j}=\left\langle\beta(\pi), e_{i}\right\rangle$. Intuitively, $s_{j}$ corresponds to the activation of $A_{i}$ that led to $s_{k}$. The states $s_{j^{\prime}}$, where $j \leq j^{\prime} \leq k$ are all of the form $\left\langle\beta(\pi), b_{1}^{\prime}, \ldots, b_{r^{\prime}}^{\prime}, u^{\prime}\right\rangle$, for some $r^{\prime} \geq 0$. Note that there may be states $s_{j^{\prime}}, j<$ $j^{\prime} \leq k$ such that $s_{j^{\prime}}=\left\langle\beta(\pi), b_{1}^{\prime}, \ldots, b_{r^{\prime}}^{\prime}, e_{i}\right\rangle$, with $r^{\prime} \geq 1$, which denote recursive entries into $A_{i}$, but which have returned before $s_{k}$. We will now be interested in $\alpha(\pi)=s_{j} \ldots s_{k}$, the suffix of $\pi$ from $s_{j}$.

What we want to do now is to project $\alpha(\pi)$ to the nodes, calls and returns in $A_{i}$, discarding fragments of runs in modules called from $A_{i}$. To do this, let 
us define a projection function $\rho_{\beta}^{i}$, for a sequence $\beta=\left\langle b_{1}, \ldots, b_{r}\right\rangle$, of any state $s$ as follows: if $s=\langle\beta, u\rangle$, where $u \in N_{i}$, then $\rho_{\beta}^{i}(s)=u$; if $s=\langle\beta, b, u\rangle$, where $(b, u) \in$ Calls $_{i} \cup$ Retns $_{i}$, then $\rho_{\beta}^{i}(s)=(b, u)$; in all other cases, $\rho_{\beta}^{i}(s)=\varepsilon$, the empty word. We extend $\rho_{\beta}^{i}$ to sequences of states: $\rho_{\beta}^{i}\left(s_{1}^{\prime} \ldots s_{l}^{\prime}\right)=\rho_{\beta}^{i}\left(s_{1}^{\prime}\right) \ldots \rho_{\beta}^{i}\left(s_{l}^{\prime}\right)$.

We can now define a function $\mu_{i}$ that extracts the local memory from the play $\pi: \mu_{i}(\pi)=\rho_{\beta(\pi)}^{i}(\alpha(\pi))$. Thus the local memory of a play $\pi$ ending in a state $s_{k}$ stands for the fragment of the play in the current module that gives the sequence of nodes, calls and returns in $A_{i}$ that led to the state $s_{k}$, ignoring the sub-plays in called modules (including recursive calls to itself).

A modular strategy $f$ for player 0 is a strategy for player 0 such that for all plays $\pi, \pi^{\prime}$ of $A$, if the control after $\pi$ and $\pi^{\prime}$ are both in $A_{i}$ and $\mu_{i}(\pi)=\mu_{i}\left(\pi^{\prime}\right)$ then $f(\pi)=f\left(\pi^{\prime}\right)$ holds. In other words, the advice of the strategy $f$ for any play $\pi$ which is currently in $A_{i}$ depends only on the local memory $\mu_{i}(\pi)$.

Since a modular strategy depends only on the local memories, we can alternatively view the modular strategy as a set of strategies, one for each game module. A strategy for a game module $A_{i}$ is a function $f_{i}:\left(N_{i} \cup \text { Calls }_{i} \cup \text { Retns }_{i}\right)^{*} \rightarrow$ $\left(N_{i} \cup\right.$ Calls $\left._{i}\right)$. A local strategy $\widehat{f}$ is $\left\{f_{i}\right\}_{i=0}^{n}$ where $f_{i}$ is a strategy for $A_{i}$. A play $\pi$ is according to $\widehat{f}$ if for every prefix $\pi^{\prime} s s^{\prime}$ of $\pi$, if the control after $\pi^{\prime} s$ is in $A_{i}$ and $s$ is in $Q_{0}$, then the following holds: let $\mu_{i}\left(\pi^{\prime} s\right)=w$, then $\mu_{i}\left(\pi s s^{\prime}\right)=w u$ where $u=f_{i}(w)$.

For a modular strategy $f$, we can associate with it a local strategy. Let $\pi$ be a play consistent with $f$. Set $\beta=\beta(\pi)$ and let the control after $\pi$ be in $A_{i}$. Then, $f_{i}\left(\mu_{i}(\pi)\right)=\rho_{\beta}^{i}(f(\pi))$ (the function $f_{i}$ on other values can be defined arbitrarily). Then it is easy to see that the plays according to $f$ are precisely the plays according to the local strategies. Conversely, given a local strategy $\widehat{f}=\left\{f_{1}, \ldots, f_{n}\right\}$, we can associate a modular strategy $f$ with it. Let $\pi s$ be a play consistent with $\widehat{f}$, let the control after $\pi s$ be in $A_{i}$, and let $\beta(\pi s)=\beta$. Then $f(\pi s)=s^{\prime}$ where $s^{\prime}$ is the unique successor of $s$ such that $\rho_{\beta}^{i}\left(s^{\prime}\right)=f_{i}\left(\mu_{i}(\pi s)\right)$. Again, it is easy to see that $f$ is modular and that the sets of plays according to $f$ and $\widehat{f}$ are the same. In the sequel, we freely switch between modular strategies and the corresponding local strategies.

We consider the following decision problem.

Given a recursive game $\left\langle A, e_{1}, X\right\rangle$, is there a modular winning strategy (or equivalently, a local strategy that is winning) for the protagonist?

Consider the game graph in Figure 1] Note that the only place where the protagonist has a choice is in picking the move from $e_{2}$. If the target set is $\left\{x_{2}^{\prime}, x_{3}^{\prime}\right\}$ then the protagonist has a winning modular strategy where it chooses to move to the exit node $x_{2}$ in $A_{2}$. For the target set $\left\{x_{1}^{\prime}, x_{3}^{\prime}\right\}$, there is no modular strategy for the protagonist that is winning. However, it is easy to see that there is a global winning strategy.

The idea behind modular strategies is that it is more appropriate to look for strategies for the modules rather than a monolithic strategy. However, rather than allowing a strategy for a module $A_{i}$ to remember only the play from the last call to $A_{i}$, we could allow the strategy to remember all parts of the play when 
it was inside $A_{i}$. That is, we could allow strategies to have a persistent memory where it is allowed to remember how the play evolved in all the previous calls to the module. For example, in the recursive game in Figure 1 though there is no modular strategy for the protagonist for the target set $\left\{x_{1}^{\prime}, x_{3}^{\prime}\right\}$, there is a persistent strategy (the strategy for $A_{2}$ picks $x_{1}$ when it is first called and picks $x_{2}$ on the second call). Checking for persistent strategies, however, turns out to be undecidable (see Section 5 for details).

From now on, when the context is clear, we use the term strategy to mean modular strategies.

\section{Solving Recursive Games}

Let us fix a reachability game $\left\langle A, e_{1}, X\right\rangle$ for the rest of this section, where $A=$ $\left\langle A_{1}, \ldots, A_{k}\right\rangle$, and each $A_{i}=\left(N_{i}, B_{i}, V_{i}^{0}, V_{i}^{1}, Y_{i}, E n_{i}, E x_{i}, \delta_{i}\right)$.

Consider $f$, a modular strategy for $\left\langle A, e_{1}, X\right\rangle$. The key to deciding recursive games is the observation that whether $f$ is winning or not is primarily determined by finding, for each $A_{i}$, the set of exit nodes of $A_{i}$ which the local strategy $f_{i}$ will lead a play entering $A_{i}$ to. Let $X_{i}^{f}$ denote the set of exits a play can reach if it enters $A_{i}$ and continues according to $f$; that is, an exit point $x \in E x_{i}$ is in $X_{i}^{f}$ if there is a play according to $f$ of the form $\pi\left\langle\bar{b}, e_{i}\right\rangle \pi^{\prime}\langle\bar{b}, x\rangle$. Since the strategy $f$ is modular, such exits $x$ are the ones for which there is a play according to $f$ of the form $\left\langle e_{i}\right\rangle \pi^{\prime \prime}\langle x\rangle$. In fact, if we take a winning strategy $\widehat{f}$ and replace an $f_{i}$ in $\widehat{f}$ with a different strategy $f_{i}^{\prime}$ which calls the same modules that $f_{i}$ calls and leads to the same set of exit nodes of $A_{i}$, then this will also be a winning strategy. This motivates the following definitions.

For a modular strategy $f$ for $\left\langle A, e_{1}, X\right\rangle$, the call graph of $f$ is $C_{f}=(V, \rightarrow, \lambda)$ such that $(V, \rightarrow)$ is a graph where:

$-V \subseteq\left\{A_{1}, \ldots, A_{n}\right\}$ is the set of all $A_{i}$ such that there is a play according to $f$ that enters $A_{i}$.

$-A_{i} \rightarrow A_{j}$ iff there is some play according to $f$ from $\left\langle e_{1}\right\rangle$ where there is a call from $A_{i}$ to $A_{j}$ (i.e. there is a play of the form $\left\langle e_{1}\right\rangle \pi\left\langle b_{1}, \ldots, b_{r}, e\right\rangle$ with $b_{r} \in B_{i}$ and $\left.Y\left(b_{r}\right)=A_{j}\right)$.

- For every $A_{i} \in V$, let $\lambda\left(A_{i}\right)=X_{i}^{f}$.

We first make a simple observation:

Lemma 1. Let $f$ be a winning strategy for $\left\langle A, e_{1}, X\right\rangle$ and let $C_{f}=(V, \rightarrow, \lambda)$ be the call graph of $f$. Then,$(V, \rightarrow)$ is acyclic.

Proof. If $f$ is a strategy whose call graph has a cycle, then one can show that there is a play according to $f$ that makes calls to the modules recursively according to the cycle in the call graph, and hence never reaches the target set. Such an $f$ will not be winning.

For a strategy $f$ on $\left\langle A, e_{1}, X\right\rangle$, we say that $f$ is hierarchic if the plays according to $f$ make no recursive calls (i.e., no play according to $f$ reaches a state of the form $s=\left\langle b_{1}, \ldots, b_{i}, u\right\rangle$, where $\exists l \in\{1, \ldots, n\}$ such that $u \in N_{l}$ and $b_{j} \in N_{l}$, for some $j \in\{1, \ldots i\})$. The following is immediate from the above lemma: 
Corollary 1. Reachability games admit only hierarchic winning strategies.

We recall that the target set $X$ is a subset of the exits of module $A_{1}$. An interesting consequence of the above result is that when we consider modular strategies, the only global states of the target set which can be reached on a winning modular strategy are $\langle x\rangle, x \in X$.

Motivated by the above lemma, we give a general definition of a call graph: a call graph is a tuple $C=(V, \rightarrow, \lambda)$ where $V \subseteq\left\{A_{1}, \ldots, A_{n}\right\},(V, \rightarrow)$ is an acyclic graph and $\lambda\left(A_{i}\right) \subseteq E x_{i}$, for each $A_{i} \in V$.

Let $C=(V, \rightarrow, \lambda)$ be a call graph for the game $\left\langle A, e_{1}, X\right\rangle$. Let $A_{i}$ be a module of the game. We now define a game graph $A_{i}^{C}$ which is a flat game graph associated with $A_{i}$ and $C$, where, intuitively, we replace each call $\left(b, e_{j}\right)$ to a module $A_{j}$ by a vertex where player 1 can take the game to any return $\left(b, x_{j}\right)$ where $x_{j}$ is in $\lambda\left(A_{j}\right)$. In other words, we are defining a game graph under the assumption that a call to a module $A_{j}$ could result in returns corresponding to $\lambda\left(A_{j}\right)$ and we want to solve the game for $A_{i}$ under these assumptions. The game graph $A_{i}^{C}$ will also prohibit any calls to modules that it is not supposed to call, in accordance with the call graph $C$.

Formally, $A_{i}^{C}$ is defined as follows: $A_{i}^{C}=\left(S_{i}, S_{i}^{0}, S_{i}^{1}, \gamma_{i}\right)$ where

$-S_{i}=N_{i} \cup$ Calls $_{i} \cup$ Retns $_{i}$.

$-S_{i}^{0}=\left(V_{i}^{0} \cap N_{i}\right) \cup\left\{(b, x) \in\right.$ Retns $\left._{i} \mid b \in V_{i}^{0}\right\}$.

$-S_{i}^{1}=\left(V_{i}^{1} \cap N_{i}\right) \cup\left\{(b, x) \in\right.$ Retns $\left._{i} \mid b \in V_{i}^{1}\right\} \cup$ Calls $_{i}$.

- The transition function $\gamma_{i}$ is defined as follows:

1. If $u \in N_{i} \cup$ Retns $_{i}, \gamma_{i}(u)=\delta_{i}(u)$.

2. If $(b, e) \in$ Calls $_{i}$ and $A_{Y(b)}$ is a successor of $A_{i}$ in $C$, then $\gamma_{i}((b, e))=\left\{(b, x) \mid x \in \lambda\left(A_{Y(b)}\right)\right\}$.

3. If $(b, e) \in$ Calls $_{i}$ and $A_{Y(b)}$ is not a successor of $A_{i}$ in $C$, then $\gamma_{i}((b, e))=\emptyset$.

The graph $A_{i}^{C}$ is thus obtained by taking the vertices as the nodes, calls and returns of $A_{i}$. The nodes and returns are partitioned into 0-nodes and 1-nodes as in $A_{i}$. Also, the calls are all deemed to be 1-nodes. The transition function follows the transition function of $A_{i}$ for nodes and returns. For a call $(b, e)$, the transition function maps it to an empty set if $A_{i}$ is not permitted to call $A_{Y(b)}$ according to the call graph $C$. Note that if a play reaches such a call, then it is maximal and hence losing for player 0 . If $A_{i}$ is permitted to call $A_{Y(b)}$, then we take the expected set of exit nodes $\lambda\left(A_{Y(b)}\right)$ from the call graph and have edges to each of the returns corresponding to these exits.

We can now state the main result for which we have developed the definitions above:

Lemma 2. There exists a winning strategy for $\left\langle A, e_{1}, X\right\rangle$ if and only if there exists a call graph $C=(V, \rightarrow, \lambda)$ such that $\lambda\left(A_{1}\right) \subseteq X$ and for every $A_{i} \in V$, player 0 wins the reachability game $\left\langle A_{i}^{C}, e_{i}, \lambda\left(A_{i}\right)\right\rangle$. 
Proof. If $f$ is a winning strategy for $\left\langle A, e_{1}, X\right\rangle$, we show that its call graph $C_{f}$ satisfies the requirements of the lemma. In fact, the strategy $f_{i}$ for $A_{i}$ itself serves as a winning strategy in $\left\langle A_{i}^{C_{f}}, e_{i}, \lambda\left(A_{i}\right)\right\rangle$, for each $A_{i}$ in the call graph $C_{f}$.

Conversely, if there is a call graph $C=(V, \rightarrow, \lambda)$ that satisfies the properties of the lemma, then the strategy for any $A_{i}^{C} \in V$ serves as a strategy for $A_{i}$ in the recursive game and these strategies in fact constitute a winning strategy.

We say that a local strategy $\left\{f_{i}\right\}_{i=1}^{n}$ is memoryless if for every pair of sequences $\sigma u, \sigma^{\prime} u \in\left(N_{i} \cup \text { Calls }_{i} \cup \text { Retns }_{i}\right)^{*}, f_{i}(\sigma u)=f_{i}\left(\sigma^{\prime} u\right)$. That is, if the local strategies' selection depends only on the current node of the play.

As a corollary to the above lemma we have:

Corollary 2. If there is winning strategy for a recursive game, then there is a local memoryless winning strategy for it.

We can now show that solving reachability games is NP-complete:

Theorem 1. Reachability on recursive (as well as hierarchic) game graphs is NP-complete.

Proof. The NP procedure works as follows. First, we guess a calling graph $C=$ $(V, \rightarrow, \lambda)$ such that $A_{1} \in V$ and $\lambda\left(A_{1}\right) \subseteq X$, where $X$ is the target set. Then, for every module $A_{i}$, we check if there is a winning strategy for player 1 in $\left\langle A_{i}^{C}, e_{i}, \lambda\left(A_{i}\right)\right\rangle$. If all the games are winning for player 0 , we report that player 0 wins the recursive game. It is easy to see that this works in polynomial time. Correctness follows from Lemma 2 .

For the lower bound, we reduce the satisfiability problem of 3-CNF formulas to solving a reachability game on hierarchic game graphs. The intuition is that there is a module for each variable, where player 0 has to pick a valuation for the variable by picking an exit point. The initial module enables player 1 to pick any clause and call a module corresponding to that clause. In the module for each clause, player 0 points to a literal that witnesses the clause satisfaction by calling the module of the corresponding variable. Player 0 wins if all clauses are satisfied. Since a modular strategy picks essentially a valuation of the variables independent of the context in which it is called, player 0 wins iff the formula is satisfiable.

\section{A Labeling Algorithm}

In this section, we describe an exponential-time realization of the nondeterministic procedure sketched in the previous section. The algorithm we present is an extension of the usual attractor set construction on flat graphs [13] adapted to our setting, and, it computes the vertices where player 0 can win in an incremental on-the-fly fashion.

Our algorithm iteratively labels vertices of a recursive game graph with tuples of sets of exit nodes according to some initialization and update rules. The 


\section{Algorithm REACH}

$\overline{\text { Initially, each exit } u} \in X$ is labeled by tuples $\left\langle E_{1}, \ldots, E_{n}\right\rangle$, where $E_{1}=\{x\}$ and $E_{i}=\top$, for every $i>1$. All the other nodes, calls, and returns are unlabeled.

Labels are updated according to the following rules:

Rule 1: For a 0 -node (or a 0 -return) $v$ of $A_{i}$, if $\left\langle E_{1}, \ldots, E_{n}\right\rangle$ labels $v^{\prime} \in$ $\delta_{i}(v)$ then add $\left\langle E_{1}, \ldots, E_{n}\right\rangle$ to labels of $v$.

Rule 2: For a 1-node (or a 1-return) $v$ of $A_{i}, \delta_{i}(v)=\left\{v_{1}, \ldots, v_{k}\right\}$, if (a) $\left\langle E_{1}^{h}, \ldots, E_{n}^{h}\right\rangle$ labels $v_{h}$ for $h=1, \ldots, k$, and (b) for every $l \neq i$, $E_{l}^{1}, \ldots, E_{l}^{k}$ are pairwise consistent, then add $\left\langle E_{1}^{\prime}, \ldots, E_{n}^{\prime}\right\rangle$ to labels of $v$, where $E_{j}^{\prime}=\bigcup_{h=1}^{k} E_{j}^{h}$ for $j=1, \ldots, n$.

Rule 3: For a return $(b, x)$ of $A_{i}$ labeled by $\left\langle E_{1}, \ldots, E_{n}\right\rangle$ where $Y_{i}(b)=j$, add $\left\langle E_{1}^{\prime}, \ldots, E_{k}^{\prime}\right\rangle$ to labels of $x$ where $E_{j}^{\prime}=\{x\}$ and $E_{l}^{\prime}=\top$ for $l \neq j$.

Rule 4: For a call $(b, e)$ of $A_{i}$ such that $Y_{i}(b)=j$, let $\left(b, x_{1}\right), \ldots,\left(b, x_{k}\right)$ be any $k$ distinct returns from $(b, e)$. Suppose that for $h=1, \ldots, k$, $\left\langle E_{1}^{h}, \ldots, E_{n}^{h}\right\rangle$ labels $\left(b, x_{h}\right)$ and $\left\langle E_{1}^{0}, \ldots, E_{n}^{0}\right\rangle$ labels $e$ in $A_{j}$. If $E_{i}^{0}=\top$, $E_{j}^{0}=\left\{x_{1}, \ldots, x_{k}\right\}$, and $E_{l}^{0}, \ldots, E_{l}^{k}$ are pairwise consistent for $l \neq i$, then add $\left\langle E_{1}, \ldots, E_{n}\right\rangle$ to labels of $(b, e)$, where $E_{l}=\bigcup_{h=0}^{k} E_{l}^{h}$ for $l=1, \ldots, n$.

The algorithm halts when there are no more labels that can be added. Then, it gives an affirmative answer if and only if $e_{1}$ is labeled with a tuple $\left\langle E_{1}, \ldots, E_{k}\right\rangle$ such that $E_{1} \subseteq X$.

Fig. 2. Algorithm REACH.

algorithm halts when the computed labeling reaches its fixed-point, i.e., there are no new labels that can be added.

Let $A=\left\langle A_{1}, \ldots, A_{n}\right\rangle$ be a recursive game graph. Consider the reachability game $\left\langle A, e_{1}, X\right\rangle$. We use a special symbol $\top$ and we overload the set union operator with the rule $\top \cup E=E$, for any set $E$. We use $v$ to denote a node, a call, or a return of $A_{j}$, for any $j$. We describe now the algorithm REACH (Figure 2) that solves the modular reachability problem. Algorithm REACH consists of labeling iteratively each $v$, with tuples of the form $\left\langle E_{1}, \ldots, E_{n}\right\rangle$, where each $E_{i}$ is either a subset of $E x_{i}$ or is the symbol $T$. Each $v$ could be labeled at any time with a set of such labels.

The reason we need to keep these labels, as opposed to just a set in the attractor-set computation, is to ensure that the strategy we construct is modular. Since the exit nodes which a strategy $f_{i}$ takes us to in $A_{i}$ is the important information we need to know, these labels will ensure that we can consistently pick a single strategy $f_{i}$ for each $A_{i}$ in the end.

For a set of maximal finite plays $\Pi$, let $\operatorname{Final}(\Pi)=\{s \mid$ there is a play of the form $\pi s \in \Pi\}$. Intuitively, $\operatorname{Final}(\Pi)$ is the exact set of states the plays in $\Pi$ end in.

When $v \in N_{i} \cup$ Calls $_{i} \cup$ Retns $_{i}$ gets a label $\left\langle E_{1}, \ldots, E_{n}\right\rangle$, it is supposed to mean that there exists a local strategy $\widehat{f}=\left\{f_{i}\right\}$ such that the following hold: 
A1. If $\Pi_{v}$ is the set of all maximal plays starting at $\langle v\rangle$ and consistent with $\widehat{f}$, then $\Pi_{v}$ contains only finite plays and $\operatorname{Final}(\Pi)=\left\{\left\langle x_{i}\right\rangle \mid x_{i} \in E_{i}\right\}$. Further, the plays in $\Pi_{v}$ do not enter any $A_{j}$, where $E_{j}=\top$, nor does it re-enter $A_{i}$.

A2. Let $l$ be such that $E_{l} \neq \top$ and $l \neq i$. Let $\Pi_{l}$ be the set of maximal plays starting at $\left\langle e_{l}\right\rangle$ and consistent with $\widehat{f}$. Then $\Pi_{l}$ consists of only finite plays and Final $(\Pi)=\left\{\left\langle x_{l}\right\rangle \mid x_{l} \in E_{l}\right\}$. Further, the plays in $\Pi_{l}$ never enter any module $A_{j}$, where $E_{j}=\top$, nor does it enter $A_{i}$.

Intuitively, when a node in $A_{i}$ is labeled with $\left\langle E_{1}, \ldots, E_{n}\right\rangle$, it signifies that there is a set of strategies for each module $A_{j}$, where $E_{j} \neq \top$, such that the strategies drive the play from the current node to some node in $E_{i}$. Moreover, these strategies never make the play enter a module $A_{j^{\prime}}$ for which strategies are not assumed (i.e. where $E_{j^{\prime}}=\top$ ). Also, the strategy for $A_{j}$ drives any play entering it to the set $E_{j}$. Finally, the strategies are guaranteed not to call $A_{i}$

For any $E^{\prime}, E^{\prime \prime} \in 2^{E x_{i}} \cup\{\top\}$, we say that $E^{\prime}$ and $E^{\prime \prime}$ are consistent if $E^{\prime}=\top$, or $E^{\prime \prime}=\top$, or $E^{\prime}=E^{\prime \prime}$.

Rule 1 makes a 0-node or 0-return $u$ inherit a label of a successor. The idea is that there is a strategy from $u$, where the protagonist picks this successor and plays then according to the strategy assured at the successor, inductively. At a 1-node or a 1-return $u$, the protagonist has no choice - it can be taken to any of the successor nodes. The rule hence labels $u$ only if all the successors agree upon the exit-nodes that they assume for all components. Rule 3 activates an exit of a module when a return corresponding to that exit is activated in some module; the activation is similar to the way we initialized our algorithm by activating the exits corresponding to the target. Calls are labeled using Rule 4: we check whether the assumptions on all modules by both the called module's entry point as well as the returns corresponding to the call are consistent, check whether the called module does not call $A_{i}$ and label the call by an appropriate label. We prove now that REACH solves the reachability problem for recursive games.

Lemma 3 (Soundness). Let $A=\left\langle A_{1}, \ldots, A_{n}\right\rangle$ be a recursive game graph. If a node, a call, or a return $v$ of $A_{i}$ is labeled by $\mathrm{REACH}$ with $\left\langle E_{1}, \ldots, E_{n}\right\rangle$, then there exists a local strategy $\widehat{f}=\left\{f_{i}\right\}$ such that (A1) and (A2) hold.

Proof. The lemma is proved by induction on the number of applications of the update rules. For the initial labeling the lemma is trivially true. The induction step is fairly straightforward from the inductive hypothesis and the intuition behind the labeling. For example, if Rule 2 is applied to label a node $u$ in $A_{i}$, then we can pick strategies for the modules from the set of strategies of its successors whose existence is guaranteed by the induction hypothesis. The only subtle point is that the selection of local strategies needs to be done carefully to ensure that the constructed strategy is not recursive (i.e. their call graph is not recursive). For Rule 2 , we pick a strategy for a module $A_{j}(j \neq i)$ as the strategy for $A_{j}$ at a successor $v^{\prime}$; however, we ensure that for every $A_{j^{\prime}}$ which is called by this strategy, we pick the strategy for $A_{j^{\prime}}$ using the strategy at $v^{\prime}$. 
Lemma 4 (Completeness). Let $A=\left\langle A_{1}, \ldots, A_{n}\right\rangle$ be a recursive game graph and $X \subseteq E x_{1}$. If there exists a winning strategy for the protagonist in the reachability game $\left\langle A, e_{1}, X\right\rangle$, then $e_{1}$ is labeled by algorithm $\mathrm{REACH}$ with $\left\langle E_{1}, \ldots, E_{n}\right\rangle$ where $E_{1} \subseteq X$.

Proof. The gist of the proof is as follows. Let $f$ be a winning strategy of the protagonist in the reachability game $\left\langle A, e_{1}, X\right\rangle$, and let $C_{f}=(V, \rightarrow, \lambda)$ be its call graph. We can then define a finite strategy tree with $f$ that encodes all the plays according to $f$ as labels of paths in the tree. One can also associate each vertex of the tree with a node in the recursive game-namely, the node reached on the play corresponding to the vertex. We then show by induction on this tree, from the leaves to the root, that for every vertex $v$ in the tree, if the node corresponding to it is $u$ in $A_{i}$, then $u$ gets a label $\left\langle E_{1}^{u}, \ldots, E_{m}^{u}\right\rangle$ such that: $E_{i} \subseteq \lambda\left(A_{i}\right)$ and for every $j \neq i, E_{j}$ is consistent with $\lambda\left(A_{j}\right)$, if $A_{j} \in V$, and $E_{j}=\top$, for every $A_{j} \notin V$. The lemma then holds from the fact that the root gets such a label.

The soundness of REACH follows from Lemma 3, by condition A1 (with $v=e_{1}$ ), while Lemma 4 proves its completeness. REACH can require exponential time since it can generate exponentially many labels. A careful analysis shows that it works in fact in time exponential in the total number of exit nodes, exponential in the maximum out-degree of the underlying graphs, but polynomial in the size of the recursive game graph. Also, note that REACH can stop once the initial node gets the appropriate label, even before reaching the fixpoint.

\section{Extensions}

Safety recursive games: Consider a recursive game graph $A$. A safety condition requires that the plays stay within a set of good vertices, or equivalently that bad vertices are avoided. A safety recursive game is $\left\langle A, e_{1}, X_{\text {good }}\right\rangle$, where $A$ is a recursive game graph, $e_{1}$ is the entry point of the game module $A_{1}$, and $X_{\text {good }}$ is a subset of $A$ nodes (let us assume that all exits are good). A play of such a game is winning if all the visited states are of the form $\left\langle b_{1}, \ldots, b_{r}, x\right\rangle$ where $x \in X_{\text {good }}$. If we restrict to modular strategies, safety recursive games are not dual to reachability recursive games. This is because in both definitions, while the protagonist is forced to use a modular strategy, the adversary is allowed to use an arbitrary strategy. In contrast with reachability, winning modular strategies in safety recursive games may not be hierarchic (in particular Lemma 1 and thus Corollary 1 do not hold). Despite this, deciding safety recursive games is also NP-complete. The hardness result is directly obtained from the reduction given in the proof of Theorem 11 For membership to NP, we can prove that the existence of a winning strategy $f$ can be witnessed by a (possibly cyclic) call graph and a tuple $\left\langle E_{1}, \ldots, E_{n}\right\rangle$, where $E_{i}$ is a subset of the exits of $A_{i}$, with the meaning that: 1 ) in each module $A_{i}$, we can visit only calls to modules that are successors in the call graph, and 2) the winning local strategy in $A_{i}$ stays within the safe set $X_{\text {good }}$ and it either never exits $A_{i}$ or it exits through an exit 
in $E_{i}$. Once this is guessed, checking that indeed a strategy is winning reduces to solving safety games on flat graphs whose total size is polynomial in the size of the recursive game graph.

Handling multiple entries: Consider a recursive game $\left\langle A, e_{1}, X\right\rangle$ where each module is allowed to have multiple entry nodes. The semantics of the game and modular strategies are the natural extensions of those for the single-entry setting. When a play enters a module, since the strategy for the module knows the entry point where the module enters, it could follow completely different strategies for the different entry nodes and still remain modular. Hence, we can replace every module which has, say, $m$ entry nodes with a set of $m$ modules, one for each entry point, but where each new module has only one entry. We suitably change the calls from other modules so that they call the corresponding modules with the appropriate entry point. It is easy to see that one can check for a modular strategy on the original game by checking for a modular strategy in this game. Consider a recursive game $G$ with $n$ modules and let $m_{e}$ be the maximum number of entries to any module. Then, it is easy to see that the above reduction produces a game graph with at most $n \cdot m_{e}$ modules and the overall size of the game graph is at most $|G|^{3}$, where $|G|$ is the size of $G$.

Recursive games with variables: In modeling programs, it is natural to have variables over a finite domain that are abstractions of actual variables and which can be passed from module to module (see, for instance, the SLAM model checker [3]). We can extend our setup to handle this by augmenting nodes with the values of variables. These variables can be local, global or passed as parameters when calls are made to other modules. If a module $A_{i}$ has $r_{i}$ input variables, $r_{o}$ output variables and $s$ internal variables, then we can model this by having $2^{r_{i}} \cdot\left|E n_{i}\right|$ entry nodes, $2^{r_{o}} \cdot\left|E x_{i}\right|$ exits, and $2^{r_{i}+r_{o}+k} \cdot\left|N_{i}\right|$ internal nodes (we assume all variables be boolean). Note that a modular strategy will be such that the strategy for a module can take into account the parameters that are sent and returned when calling the module, but cannot know the exact evolution of the of the called program. This makes our setting a very natural setup where we can deal with the construction of skeletons of program modules which achieve a particular specification.

Persistent memory strategies: As mentioned in Section 2.3, we can relax the condition of modular strategies and instead ask for a persistent strategy, where a strategy for a module can remember not only the play from the last call to the module, but all parts of the play when the play entered into this module. The idea is that we can realize the strategy for a module as a program which has a static memory to store all that happens within the module, and use this information to drive the play. However, it turns out that checking whether there is a persistent strategy in a given recursive game is undecidable. The reduction is from the undecidability of solving multi-player games with incomplete information [14. We have:

Theorem 2. The problem of checking whether there is a winning persistent strategy in a given hierarchical game is undecidable. 


\section{References}

1. R. Alur, K. Etessami, and M. Yannakakis. Analysis of recursive state machines. In Proc. of the 13th International Conference on Computer Aided Verification, $C A V^{\prime} 01$, LNCS 2102, pages 207-220. Springer, 2001.

2. R. Alur, T. Henzinger, and O. Kupferman. Alternating-time temporal logic. Journal of the ACM, 49(5):1-42, 2002.

3. T. Ball and S. Rajamani. Bebop: A symbolic model checker for boolean programs. In Proceedings of the SPIN 2000 Workshop on Model Checking of Software, LNCS 1885, pages 113-130. Springer, 2000.

4. A. Bouajjani, J. Esparza, and O. Maler. Reachability analysis of pushdown automata: Application to model-checking. In Proc. 8th Conference on Concurrency Theory, volume 1243 of $L N C S$, pages 135-150, Warsaw, July 1997. Springer.

5. J. Büchi and L. Landweber. Solving sequential conditions by finite-state strategies. Trans. AMS, 138:295-311, 1969.

6. T. Cachat. Symbolic strategy synthesis for games on pushdown graphs. In $A u$ tomata, Languages and Programming, 29th Int'l Coll., ICALP, Malaga, Spain, July 8-13, 2002, Proceedings, volume 2380 of $L N C S$, pages 704-715. Springer.

7. A. Chakrabarti, L. de Alfaro, T. Henzinger, M. Jurdzinski, and F. Mang. Interface compatibility checking for software modules. In Proceedings of the 14 th Int'l Conf. on Computer-Aided Verification, LNCS 2404, pages 428-441. Springer, 2002.

8. L. de Alfaro and T. A. Henzinger. Interface automata. In Proceedings of the Ninth Annual Symposium on Foundations of Software Engineering (FSE), pages 109-120. ACM Press, 2001.

9. E. A. Emerson. Model checking and the mu-calculus. In N. Immerman and P. Kolaitis, editors, Proceedings of the DIMACS Symposium on Descriptive Complexity and Finite Models, pages 185-214. American Mathematical Society Press, 1997.

10. O. Kupferman and M. Vardi. Church's problem revisited. The Bulletin of Symbolic Logic, 5(2):245-263, June 1999.

11. O. Kupferman, M. Vardi, and P. Wolper. Module checking. Information and Computation, 164(2):322-344, 2001.

12. P. Madhusudan and P. S. Thiagarajan. A decidable class of asynchronous distributed controllers. In Proceedings of the 13th International Conference on Concurrency Theory (CONCUR '02), LNCS 2421, pages 145-160. Springer, 2002.

13. R. McNaughton. Infinite games played on finite graphs. Annals of Pure and Applied Logic, 65:149-184, 1993.

14. G. Peterson and J. Reif. Multiple-person alternation. In Proc. 20st IEEE Symposium on Foundation of Computer Science, pages 348-363, 1979.

15. A. Pnueli and R. Rosner. On the synthesis of a reactive module. In Proc. 16th ACM Symposium on Principles of Programming Languages, Austin, January 1989.

16. A. Pnueli and R. Rosner. Distributed reactive systems are hard to synthesize. In Proc. 31st IEEE Symposium on Foundation of Computer Sc., pages 746-757, 1990.

17. K. Rudie and W. Wonham. Think globally, act locally: Decentralized supervisory control. IEEE Transactions on Automatic Control, 37(11):1692-1708, 1992.

18. W. Thomas. Languages, automata, and logic. Handbook of Formal Language Theory, III:389-455, 1997.

19. W. Thomas. Infinite games and verification. In Proceedings of the International Conference on Computer Aided Verification CAV'02, volume 2404 of Lecture Notes in Computer Science, pages 58-64. Springer, 2002.

20. I. Walukiewicz. Pushdown processes: Games and model-checking. Information and Computation, 164(2):234-263, January 2001. 